\title{
Disease-Specific Clinical Trial Networks: the Example of Cystic Fibrosis
}

Cystic Fibrosis (CF) is an autosomal recessive, life-shortening multiorgan disorder. It is the most frequent rare disease affecting about 42.000 patients in Europe and more than 80.000 patients worldwide. CF is caused by mutations in the Cystic Fibrosis Transmembrane Conductance Regulator (CFTR) gene leading to absent or defective chloride channels (CFTR).

Meanwhile, more than half of the CF population has reached adulthood. Factors that have contributed to this milestone include structured follow-up by dedicated, multi-disciplinary care teams in specialized CF centres and by utilizing optimal standard of care treatments right after diagnosis. Although steady progress in survival was achieved, the median age of death is still 28 years [1].

Novel therapies are being developed targeting the basic defect; however, they do not yet provide a cure for the disease and are administered in addition to symptomatic treatments. The ultimate goal remains to restore CFTR function in every CF patient independent of genotype and to prevent disease manifestations by treating the basic defect of CF immediately after a confirmed diagnosis.

The rationale is to optimize the development and evaluation of new and approved treatments for CF through efficient clinical studies in Europe. This includes advising on optimal study design, identifying the most appropriate target population, improving sample size calculations by using real life data (including data from the ECFS CF Patient Registry) and decreasing the sample size needed by standardization of outcome parameters. Apart from study design, motivating patients to take part in research and promoting the safety of participants in clinical trials are of great importance.

To establish a best strategy to design and implement clinical trials in CF a disease specific clinical trials network was founded in Europe in 2009 (European Cystic Fibrosis Society - Clinical Trials Network; ECFS-CTN) [2].
Clinical trial networks provide a centralized resource for the successful execution of a clinical trial. For diseases with relatively small populations such as CF, the most appropriate action is to form a larger operational group and to focus on both study quality and quantity. National initiatives towards organized CF research have been underway in Europe for some time. But many studies need inclusion numbers that surpass the capacity of national patient groups, hence the need for a European wide initiative.

The ECFS and EuroCareCF (EU Framework 6 Program) worked together to launch the ECFS-CTN. The network originally provided access to 18 large and experienced CF centres, located in 8 different countries throughout Europe. Twelve more sites were added in 2012. In 2015, 13 additional sites have been selected leading to
43 sites in 15 countries ( $\bullet$ Fig. 1 ). The network will continue to grow: a further expansion is planned in 2019/2020.

All centres fulfill a certain set of criteria such as number of patients, human resources, experience in clinical trials and infrastructure support.

The executive committee (EC) steers the activities via a coordinating centre ( $\triangleright$ Fig. 2). The EC develops and adapts the global strategies of the network. It is chaired by the CTN director who also presides the two weekly teleconferences with the preplanned agenda. Members of the EC are elected for a 3 year term and represent different European countries.

Twice a year all partners in the network meet face-to-face. This steering committee includes one investigator from each

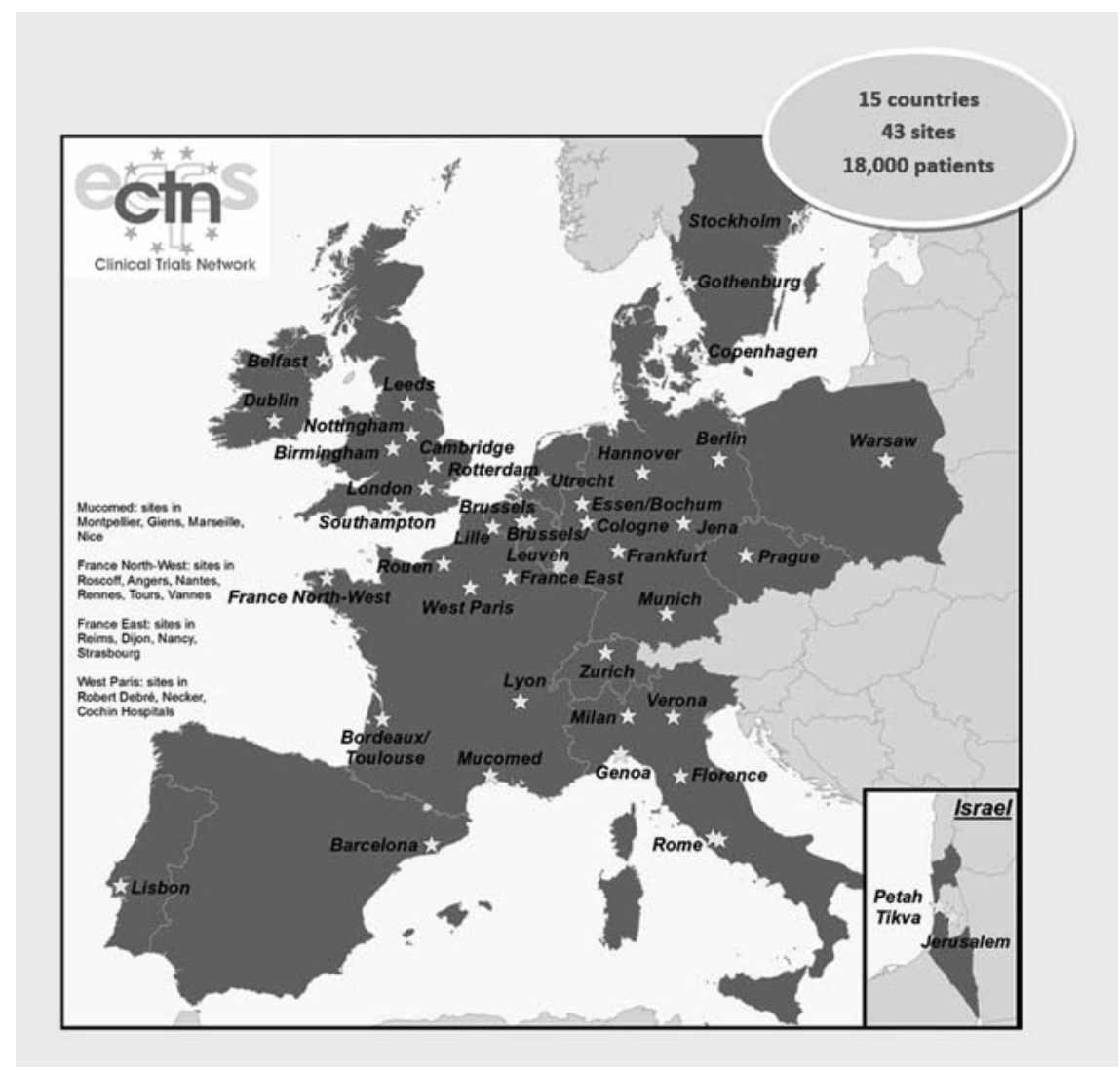

- Fig. 1 European Cystic Fibrosis Society - Clinical Trials Network (ECFS-CTN) site locations in 2018: 43 sites in 15 countries (www.ecfs-ctn.eu). 


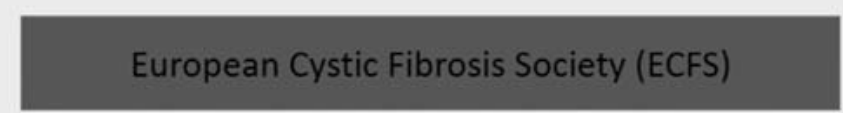

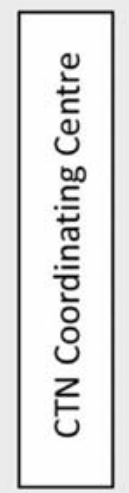

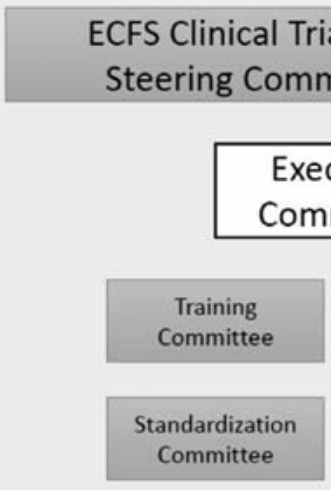

ECFS Clinical Trials Network (CTN) Steering Committee (trial sites)

\section{Executive \\ Committee}

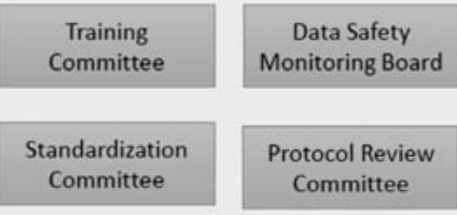

Fig. 2 Structure of the ECFS-CTN [3]

\section{Standardization Committee}

The aim of the ECFS-CTN Standardization Committee is to harmonize clinical outcome parameters used in CF clinical research by implementing Standard Operating Procedures (SOP) for the main endpoints used in clinical trials. This will ensure less variability in results and, therefore, treatment benefits can be evaluated in a more reliable way. Additionally, a lower patient number will need to be included in the trial to reach the same statistical power. The committee works closely together with the CFF-TDN on development of global SOPs.

Implementation of ECFS-CTN SOPs is validated by a processs of site certification. Outcome parameters that have been standardized and certified via central reading facilities are lung clearance index (LCI), Chest CT, nasal potential difference (NPD), and intercurrent measurements (ICM).

Different standardization working groups have been formed for inflammatory markers, lung imaging, microbiological explorations, sweat testing, nutritional evaluation, respiratory functions and patient reported outcomes.

\section{Training Committee}

As a network code of conduct all sites have agreed by being part of the network to only perform international clinical trials that have been reviewed and approved by the ECFS-CTN. Since the beginning 89 protocols have been reviewed and 73 trials supported. The protocols have been submitted at an earlier stage over the last years and the impact on protocol development has massively increased. In the long term, improvement of CF protocol quality and feasibility will bring efficient medicines faster to the patients.

Protocol feasibility assessment can take place in parallel to protocol review. The clinical trial sites provide accurate number of patients being eligible for a particular trial by consulting their patient database, which will help for appropriate site selection.
The training committee is promoting the improvement of knowledge and skills in clinical research of all investigators and research coordinators. This is achieved by organizing yearly training meetings with topics suggested by the trial sites including theoretical as well as practical training sessions.

\section{Data Safety Monitoring Board (DSMB)}

The ECFS-CTN can provide assistance for the setup of an independent Data Monitoring Safety Board with CF experts. The DSMB execute services independently from CTN. 


\section{Networking}

The purpose of the ECFS-CTN networking is to build active links between CTN and key partners involved in clinical trials in CF. This way existing resources and available expertise is used, policies are streamlined and impact is increased.

The executive committee discusses gaps or issues in CF research with representatives of the EMA (European Medicines Agency), e.g. medicines for children, the need for defining appropriate outcome parameters, etc. The objective is to improve the context for regulatory decisions in the field of CF drug development in Europe. This is achieved by providing scientifically sound and convincing information to the EMA and applicable national competent authorities thereby helping these institutions to set up appropriate guidance documents and to discuss specific applications for marketing authorization related to CF.

The ECFS-CTN is also a voting member of the coordinating group of the EMA's European Network of Paediatric Research (EnprEMA) - a network of existing national and European networks, investigators and centres with specific expertise in the performance of studies in the paediatric population.

The ECFS-CTN is partnering in several EU funded projects (e.g. European Reference Network, IMI, H2020, C4C).

\section{Quality Assurance and Sustainability}

The ECFS-CTN sites have access to the yearly report on study performance including patient enrolment in trials, other clinical trial metrics and site responsiveness to various CTN queries. The scores are provided in an anonymized way allowing the sites to benchmark their performance against other sites. Those assessments help the sites to identify areas for further improvement.

\section{Partnership with Patient Organisations (POs)}

Representatives of POs are part of the EC, the steering committees and protocol review teams. The network involves patients and POs because active clinical trial enrolment is only possible via empowerment of the patients. Patient empowerment is also promoted by brochures in different languages explaining different aspects of clinical trials. Leaflets specific for children are also available. Additionally the interest in clinical trials is boostered by educational leaflets explaining the development of their current medications.

This presentation describes the steps of the development and the structure of a disease-specific clinical trial network which is highly important in rare diseases. Activities such as reviewing study protocols, performing feasibility assessments as well as standardization and training of outcome parameters help to bring high-quality clinical trials to the patients resulting in optimization of the development of new treatments for CF. Cooperation with the pharmaceutical industry, other research networks, patient organisations and regulatory agencies is very important throughout all the activities.

The ECFS-CTN facilitates the development of new treatments for a rare disease and could be a prototype for other (rare) diseases.

\section{Conflict of Interest}

The authors declares that she has no conflict of interest.

Author

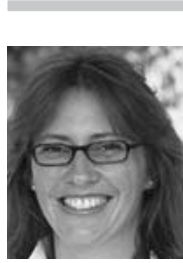

Silke van KoningsbruggenRietschel

Mukoviszidose-Zentrum Köln, Pädiatrische Pneumologie und Allergologie, Klinik und Poli-

klinik für Kinder- und Jugendmedizin, Universität zu Köln

\section{Correspondence}

Dr. Silke van Koningsbruggen-Rietschel Mukoviszidose-Zentrum Köln

Pädiatrische Pneumologie und Allergologie Klinik und Poliklinik für Kinder- und Jugendmedizin

Universität zu Köln

Kerpener Str. 62

50937 Köln

Silke.vanKoningsbruggen@uk-koeln.de

Director

ECFS-CTN

UZ Gasthuisberg

Herestraat 49

3000 Leuven

Belgium

ECFS-CTN@uzleuven.be

\section{References}

[1] Zolin A, McKone EF, van Rens J et al. ECFS Patient Registry Annual Data Report 2010. Karup: European Cystic Fibrosis Society; 2014

[2] De Boeck K, Bulteel V, Tiddens $\mathrm{H}$ et al.; on behalf of all ECFS-CTN network partners. Guideline on the design and conduct of cystic fibrosis clinical trials: The European Cystic Fibrosis Society - Clinical Trials Network (ECFS-CTN). JCF 2011; 10 (Suppl. 2): S67-S74

[3] Boeck K, Bulteel V, Fajac, I. Disease-specific clinical trials networks: the example of cystic fibrosis. Eur J Pediatr 2016; 175: 817-824 